\title{
V2X communication system with non-orthogonal multiple access: outage performance perspective
}

\author{
Tu-Trinh Thi Nguyen, Dinh-Thuan Do
}

Faculty of Electronics Technology, Industrial University of Ho Chi Minh City (IUH), Ho Chi Minh City, Vietnam

\begin{tabular}{|c|c|}
\hline Article Info & ABSTRACT \\
\hline Article history: & \multirow{9}{*}{$\begin{array}{l}\text { To achieve low-latency and high-reliability (LLHR) for applications in the vehicle- } \\
\text { to-everything (V2X) networks, the non-orthogonal multiple access (NOMA) is pro- } \\
\text { posed for Long Term Evolution (LTE)as a promising technology. NOMA-V2X pro- } \\
\text { vides higher spectrum efficiency compared with the orthogonal multiple access (OMA) } \\
\text { based V2X. The vehicles are expected to serve different services with variety of data } \\
\text { transmission. The cluster of vehicles could be grouped to achieve better service from } \\
\text { the transmitter sources. This study presents two-way relay assisted NOMA-V2X trans- } \\
\text { mission by exploiting amplify-and-forward (AF) and full-duplex technique. We can } \\
\text { benefits from potential applications of NOMA-V2X system with respect to serving } \\
\text { massive users and adapting higher bandwidth efficiency. We derive expressions of } \\
\text { outage probability to evaluate performance of two vehicles and to improve the quality } \\
\text { of service (QoS) for the device with the poor channel conditions. The main investi- } \\
\text { gation related two users' performance which provides guidelines to design practical } \\
\text { system. These expressions are further verified by Monte-Carlo simulations. }\end{array}$} \\
\hline Received Jun 9, 2020 & \\
\hline Revised Sep 11, 2020 & \\
\hline Accepted Sep 25, 2020 & \\
\hline Keywords: & \\
\hline & \\
\hline access & \\
\hline Outage probability & \\
\hline Vehicle-to-everything & \\
\hline
\end{tabular}

This is an open access article under the CC BY-SA license.

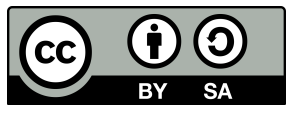

Corresponding Author:

Dinh-Thuan Do,

Faculty of Electronics Technology,

Industrial University of Ho Chi Minh City (IUH),

Ho Chi Minh City, Vietnam.

Email: dodinhthuan@iuh.edu.vn

\section{INTRODUCTION}

As a candidate technique for forthcoming $5 \mathrm{G}$ networks, non-orthogonal multiple access (NOMA) network has recently drawn in considerable attentions [1]-[8]. In particular, the higher spectral efficiency benefits can be achieved by the deployment of NOMA and it outperforms the traditional orthogonal multiple access (OMA) scheme [1]-[3]. In practical NOMA systems, to achieve low complexity, the successive interference cancellation (SIC) decoding technique is needed to satisfy the performance of the NOMA system and it requires the user grouping as an important issue. In the recent works regarding NOMA system, the improved performance can be achieved as by employing relaying networks [4]-[7], [9]-[13] with NOMA to introduce new paradigm termed as cooperative NOMA [8]. Regarding system performance, the optimal sum rate [14], [15], and the minimal transmit power [16] are introduced with respect to the user grouping.

Recently, to provide smarter, safer and more efficient road traffic, vehicle-to-everything (V2X) communications have a lot of achievements in both academia and industry [16]-[18]. Three kinds of V2X networks including vehicle-to-vehicle (V2V), vehicle-to-infrastructure (V2I) and vehicle-to-pedestrian (V2P) are implemented to enable real-time traffic information exchange among infrastructure, vehicles, and pedestrians [19]-[21]. In such a V2X, low access efficiency and data congestion are caused by the fast growth of number 
of connected vehicles. The development of V2X communications need be tackled the challenges in vehicular networks such as [22], [23]. This paper considers the ability of two pairs of vehicle can be communicated via the Roadside Unit (RSU).

\section{NETWORK ARCHITECTURE AND PROTOCOL DESCRIPTIONS}

\subsection{System architecture}

Figure 1 depicts a scenario of AF relay assisted two-way NOMA-V2X systems, all nodes are with single antenna except for relay with two antennas for FD mode. The base stations (BSs) employ a RSU to serve group of two vehicles. In the multicasting scenario, RSU serves multiple users using NOMA. In this case, vehicles in the same group require to receive different information (e.g., vehicle-specific control information) from the BS. The source $S_{1}, S_{2}$ are able to send the corresponding signals $x_{1}, x_{2}$ (with power allocation factors $\left.\alpha_{1}, \alpha_{2}\right)$ to the RSU in the same time. The constraint of power allocation factors, i.e., $\alpha_{1}>\alpha_{2}$ and $\alpha_{1}+\alpha_{2}=1$. It can be shared by two source-destination Group $1=\left\{\mathrm{S}_{1}, \mathrm{U}_{1}\right\}$ and Group2 $=\left\{\mathrm{S}_{2}, \mathrm{U}_{2}\right\}$ pairs. The transmit power at sources $S_{1}, S_{2}$ are the same, i.e. equals to $P_{s}$. The channels for links $S_{1}$-RSU, $S_{2}-\mathrm{RSU}, \mathrm{RSU}-U_{1}$ and RSU- $U_{2}$ are $h_{1}, h_{2}, g_{1}, g_{2}$.

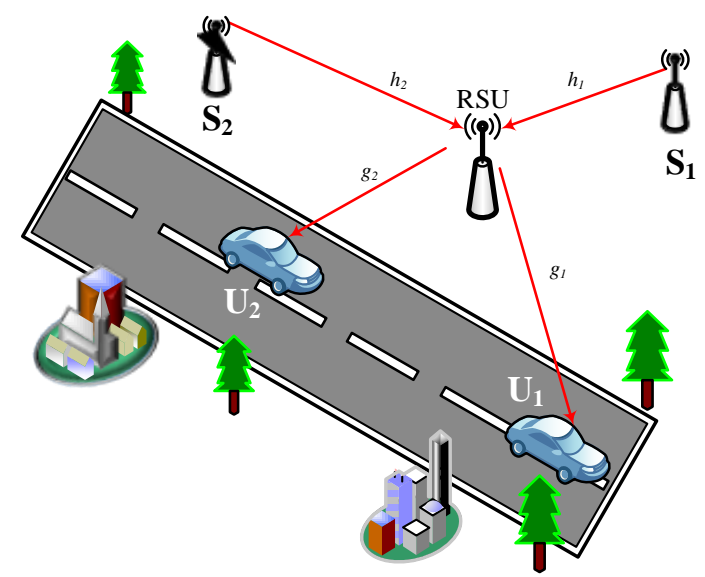

Figure 1. System model of NOMA V2X

\subsection{SINR calculation}

The received signal at RSU is given by

$$
y_{\mathrm{RSU}}=\sqrt{\alpha_{1} P_{s}} h_{1} x_{1}+\sqrt{\alpha_{2} P_{s}} h_{2} x_{2}+P_{\mathrm{RSU}} f+\eta_{\mathrm{RSU}}
$$

where $\eta_{\mathrm{RSU}}$ is AWGN noise with variance of $\sigma_{0}^{2}$. We call $f$ as self-channel due to FD mode applied at the RSU. The received signal at $\mathrm{U}_{\mathrm{i}},(\mathrm{i}=1,2)$ can be expressed as follows:

$$
y_{\mathrm{U}_{\mathrm{i}}}=\sqrt{P_{\mathrm{RSU}}} \beta g_{\mathrm{i}}\left(\sqrt{\alpha_{1} P_{s}} h_{1} x_{1}+\sqrt{\alpha_{2} P_{s}} h_{2} x_{2}+\sqrt{P_{\mathrm{RSU}}} f+\eta_{\mathrm{RSU}}\right)+\eta_{\mathrm{U}_{\mathrm{i}}} .
$$

The amplify factor is defined as follows:

$$
\beta=\frac{1}{\sqrt{\alpha_{1} P_{s}\left|h_{1}\right|^{2}+\alpha_{2} P_{s}\left|h_{2}\right|^{2}+P_{\mathrm{RSU}}|f|^{2}+\sigma_{0}^{2}}},
$$

where $\rho_{s} \triangleq P_{s} / \sigma_{0}^{2}$ and $\rho_{\mathrm{RSU}} \triangleq P_{\mathrm{RSU}} / \sigma_{0}^{2}$ are transmission SNR of source and relay node, respectively. 
The SINR at destination 1 in order to decode its own data can be written by

$$
\gamma_{\mathrm{U}_{1}}=\frac{\alpha_{1} \rho_{s} \rho_{\mathrm{RSU}}\left|g_{1}\right|^{2}\left|h_{1}\right|^{2}}{\alpha_{2} \rho_{s} \rho_{\mathrm{RSU}}\left|g_{1}\right|^{2}\left|h_{2}\right|^{2}+\rho_{\mathrm{RSU}}\left|g_{1}\right|^{2}+\alpha_{1} \rho_{s}\left|h_{1}\right|^{2}+\rho_{\mathrm{RSU}} \rho_{\mathrm{RSU}}|f|^{2}\left|g_{1}\right|^{2}+1} .
$$

The received SINR for destination 2 to decode message $x_{1}$ is given by

$$
\gamma_{\mathrm{U}_{2} \rightarrow 1}=\frac{\alpha_{1} \rho_{\mathrm{RSU}} \rho_{s}\left|g_{2}\right|^{2}\left|h_{1}\right|^{2}}{\alpha_{2} \rho_{\mathrm{RSU}} \rho_{s}\left|h_{2}\right|^{2}\left|g_{2}\right|^{2}+\rho_{\mathrm{RSU}}\left|g_{2}\right|^{2}+\alpha_{1} \rho_{s}\left|h_{1}\right|^{2}+\rho_{\mathrm{RSU}} \rho_{\mathrm{RSU}}|f|^{2}\left|g_{2}\right|^{2}+1} .
$$

Destination 2 detects its own message with the following SINR.

$$
\gamma_{\mathrm{U}_{4}}=\frac{\alpha_{2} \rho_{\mathrm{RSU}} \rho_{s}\left|h_{2}\right|^{2}\left|g_{2}\right|^{2}}{\rho_{\mathrm{RSU}}\left|g_{2}\right|^{2}+\alpha_{1} \rho_{s}\left|h_{1}\right|^{2}+\rho_{\mathrm{RSU}} \rho_{\mathrm{RSU}}|f|^{2}\left|g_{2}\right|^{2}+1}
$$

\section{OUTAGE AND THROUGHPUT PERFORMANCE ANALYSIS}

\subsection{The outage probability for group 1}

The outage probability is basic metric showing probability to SINR less than threshold value. In particular, the outage probability of group 1 can be written as:

$$
\mathrm{OP}_{\Sigma_{1}}^{\mathrm{FD}}=\operatorname{Pr}\left(\gamma_{\mathrm{U}_{1}}<\gamma_{0}^{1}\right)
$$

Next, $\mathrm{OP}_{\Sigma_{1}}^{\mathrm{FD}}$ can be computed as

$$
\begin{aligned}
\mathrm{OP}_{\Sigma_{1}}^{\mathrm{FD}} & =\operatorname{Pr}\left(\frac{\alpha_{1} \rho_{s} \rho_{\mathrm{RSU}}\left|g_{1}\right|^{2}\left|h_{1}\right|^{2}}{\alpha_{2} \rho_{s} \rho_{\mathrm{RSU}}\left|g_{1}\right|^{2}\left|h_{2}\right|^{2}+\rho_{\mathrm{RSU}}\left|g_{1}\right|^{2}+\alpha_{1} \rho_{s}\left|h_{1}\right|^{2}+\rho_{\mathrm{RSU}} \rho_{\mathrm{RSU}}|f|^{2}\left|g_{1}\right|^{2}+1}<\gamma_{0}^{1}\right) \\
& =\operatorname{Pr}\left(1,\left|g_{1}\right|^{2} \leq \frac{\gamma_{0}^{1}}{\rho_{\mathrm{RSU}}}\right)+\operatorname{Pr}\left(\left|h_{1}\right|^{2}<\frac{\rho_{\mathrm{RSU}}\left|g_{1}\right|^{2}\left(\alpha_{2} \rho_{s}\left|h_{2}\right|^{2}+\rho_{\mathrm{RSU}}|f|^{2}+1\right)+1}{\alpha_{1} \rho_{s}\left(\frac{\rho_{\mathrm{RSU}}}{\gamma_{0}^{1}}\left|g_{1}\right|^{2}-1\right)},\left|g_{1}\right|^{2} \frac{\gamma_{0}^{1}}{\rho_{\mathrm{RSU}}}\right) \\
& =1-\frac{1}{\lambda_{g_{1}}} \int_{\frac{\gamma_{0}^{1}}{\rho_{\mathrm{RSU}}}}^{\infty} \exp \left(-\frac{\rho_{\mathrm{RSU}} x X}{\lambda_{h_{1}} \alpha_{1} \rho_{s}\left(\frac{\rho_{\mathrm{RSU}}}{\gamma_{0}^{1}} x-1\right)}-\frac{1}{\lambda_{g_{1}}}-\frac{1}{\lambda_{h_{1}} \alpha_{1} \rho_{s}\left(\frac{\rho_{\mathrm{RSU}}}{\gamma_{0}^{1}} x-1\right)}\right) d x
\end{aligned}
$$

Putting $t=\frac{\rho_{\mathrm{RSU}}}{\gamma_{0}^{1}} x-1 \Rightarrow x=\frac{\gamma_{0}^{1}}{\rho_{\mathrm{RSU}}}(t+1)$ then $\mathrm{OP}_{\Sigma_{1}}^{\mathrm{FD}}$ has become

$$
\begin{aligned}
\mathrm{OP}_{\Sigma_{1}}^{\mathrm{FD}}= & 1-\frac{\gamma_{0}^{1}}{\rho_{\mathrm{RSU}} \lambda_{g_{1}}} \exp \left(-\frac{\gamma_{0}^{1}}{\lambda_{g_{1}} \rho_{\mathrm{RSU}}}-\frac{\gamma_{0}^{1} X}{\lambda_{h_{1}} \alpha_{1} \rho_{s}}\right) \\
& \times \int_{0}^{\infty} \exp \left(-\left(\frac{1}{\lambda_{h_{1}} \alpha_{1} \rho_{s}}+\frac{\gamma_{0}^{1} X}{\lambda_{h_{1}} \alpha_{1} \rho_{s}}\right) \frac{1}{t}-\frac{\gamma_{0}^{1} t}{\lambda_{g_{1}} \rho_{\mathrm{RSU}}}\right) d t \\
= & 1-\exp \left(-\frac{\gamma_{0}^{1}}{\lambda_{g_{1}} \rho_{\mathrm{RSU}}}-\frac{\gamma_{0}^{1} X}{\lambda_{h_{1}} \alpha_{1} \rho_{s}}\right) 2 \sqrt{\frac{\gamma_{0}^{1}}{\lambda_{g_{1}} \rho_{\mathrm{RSU}}}\left(\frac{1}{\lambda_{h_{1}} \alpha_{1} \rho_{s}}+\frac{\gamma_{0}^{1} X}{\lambda_{h_{1}} \alpha_{1} \rho_{s}}\right)} \\
& \times K_{1}\left(2 \sqrt{\frac{\gamma_{0}^{1}}{\lambda_{g_{1}} \rho_{\mathrm{RSU}}}\left(\frac{1}{\lambda_{h_{1}} \alpha_{1} \rho_{s}}+\frac{\gamma_{0}^{1} X}{\lambda_{h_{1}} \alpha_{1} \rho_{s}}\right)}\right)
\end{aligned}
$$


where $X=\left(\alpha_{2} \rho_{s}\left|h_{2}\right|^{2}+\rho_{\mathrm{RSU}}|f|^{2}+1\right)$. Let $Y=\alpha_{2} \rho_{s}\left|h_{2}\right|^{2}+\rho_{\mathrm{RSU}}|f|^{2} \Rightarrow X=Y+1$ then the outage probability of group 1 written again as follows

$$
\begin{aligned}
& \mathrm{OP}_{\Sigma_{1}}^{\mathrm{FD}}(Y)=1-\mathrm{e}^{-\frac{\gamma_{0}^{1}}{\lambda_{g_{1}} \rho_{r}}-\frac{\gamma_{0}^{1}(Y+1)}{\lambda_{h_{1}} \alpha_{1} \rho_{s}}} 2 \sqrt{\frac{\gamma_{0}^{1}}{\lambda_{g_{1}} \rho_{\mathrm{RSU}}}\left(\frac{1}{\lambda_{h_{1}} \alpha_{1} \rho_{s}}+\frac{\gamma_{0}^{1}(Y+1)}{\lambda_{h_{1}} \alpha_{1} \rho_{s}}\right)} \\
& \times K_{1}\left(2 \sqrt{\frac{\gamma_{0}^{1}}{\lambda_{g_{1}} \rho_{\mathrm{RSU}}}\left(\frac{1}{\lambda_{h_{1}} \alpha_{1} \rho_{s}}+\frac{\gamma_{0}^{1}(Y+1)}{\lambda_{h_{1}} \alpha_{1} \rho_{s}}\right)}\right) \\
& =1-\mathrm{e}^{-\frac{\gamma_{0}^{1} Y}{\lambda_{h_{1}} \alpha_{1} \rho_{s}}-\frac{\gamma_{0}^{1}}{\lambda_{g_{1}} \rho_{\mathrm{RSU}}}-\frac{\gamma_{0}^{1}}{\lambda_{h_{1}} \alpha_{1} \rho_{s}}} 2 \sqrt{\frac{\gamma_{0}^{1}}{\lambda_{g_{1}} \rho_{\mathrm{RSU}}}\left(\frac{\gamma_{0}^{1} Y}{\lambda_{h_{1}} \alpha_{1} \rho_{s}}+\frac{1}{\lambda_{h_{1}} \alpha_{1} \rho_{s}}+\frac{\gamma_{0}^{1}}{\lambda_{h_{1}} \alpha_{1} \rho_{s}}\right)} \\
& \times K_{1}\left(2 \sqrt{\frac{\gamma_{0}^{1}}{\lambda_{g_{1}} \rho_{\mathrm{RSU}}}\left(\frac{\gamma_{0}^{1} Y}{\lambda_{h_{1}} \alpha_{1} \rho_{s}}+\frac{1}{\lambda_{h_{1}} \alpha_{1} \rho_{s}}+\frac{\gamma_{0}^{1}}{\lambda_{h_{1}} \alpha_{1} \rho_{s}}\right)}\right) \\
& =1-2 \mathrm{e}^{-\vartheta_{1} Y-\vartheta_{2}} \sqrt{\vartheta_{3} Y+\vartheta_{4}} K_{1}\left(2 \sqrt{\vartheta_{3} Y+\vartheta_{4}}\right)
\end{aligned}
$$

where $\vartheta_{1}=\frac{\gamma_{0}^{1}}{\lambda_{h_{1}} \alpha_{1} \rho_{s}}, \vartheta_{2}=\frac{\gamma_{0}^{1}}{\rho_{\mathrm{RSU}} \lambda_{g_{1}}}+\frac{\gamma_{0}^{1}}{\alpha_{1} \rho_{s} \lambda_{h_{1}}}, \vartheta_{3}=\frac{\gamma_{0}^{1} \gamma_{0}^{1}}{\alpha_{1} \rho_{s} \rho_{\mathrm{RSU}} \lambda_{g_{1}} \lambda_{h_{1}}}, \vartheta_{4}=\frac{\gamma_{0}^{1}}{\lambda_{g_{1}} \rho_{\mathrm{RSU}}}\left(\frac{1}{\lambda_{h_{1}} \alpha_{1} \rho_{s}}+\frac{\gamma_{0}^{1}}{\lambda_{h_{1}} \vartheta_{1} \rho_{s}}\right)$. $\mathrm{OP}_{\Sigma_{1}}^{\mathrm{FD}}$ can be calculated as

$$
\begin{aligned}
\mathrm{OP}_{\Sigma_{1}}^{\mathrm{FD}} & =E_{Y}\left\{1-2 \mathrm{e}^{-\vartheta_{1} Y-\vartheta_{2}} \sqrt{\vartheta_{3} Y+\vartheta_{4}} K_{1}\left(2 \sqrt{\vartheta_{3} Y+\vartheta_{4}}\right)\right\} \\
& =\int_{0}^{\infty}\left(1-2 \mathrm{e}^{-\vartheta_{1} Y-\vartheta_{2}} \sqrt{\vartheta_{3} Y+\vartheta_{4}} K_{1}\left(2 \sqrt{\vartheta_{3} Y+\vartheta_{4}}\right)\right) f_{Y}(y) d y \\
& =\mathrm{OP}_{\Sigma_{1} 1}^{\mathrm{FD}}+\mathrm{OP}_{\Sigma_{1}, 2}^{\mathrm{FD}}
\end{aligned}
$$

We have

$$
\begin{aligned}
F_{Y}(y) & =\int_{0}^{Y} f_{Y}(y) d y \\
& =\frac{1}{\lambda_{f}} \int_{0}^{\frac{y}{\rho_{\mathrm{RSU}}}} \mathrm{e}^{-\frac{x}{\lambda_{f}}} d x-\frac{1}{\lambda_{f}} \mathrm{e}^{-\frac{y}{\lambda_{h_{2}} v_{2} \rho_{s}}} \int_{0}^{\frac{y}{\rho_{\mathrm{RSU}}}} \mathrm{e}^{-\left(\frac{1}{\lambda_{f} \rho_{r}}-\frac{1}{\lambda_{h_{2}} \alpha_{2} \rho_{s}}\right) x} d x .
\end{aligned}
$$

Here we look at two cases for CDF and PDF:

\subsubsection{Case 1}

If $\frac{1}{\rho_{\mathrm{RSU}} \lambda_{f}}-\frac{1}{\lambda_{h_{2}} \alpha_{2} \rho_{s}} \neq 0$, we have

$$
\begin{gathered}
F_{Y}(y)=1-\frac{\rho_{\mathrm{RSU}} \lambda_{f}}{\rho_{\mathrm{RSU}} \lambda_{f}-\alpha_{2} \rho_{s} \lambda_{h_{2}}} \mathrm{e}^{-\frac{y}{\rho_{\mathrm{RSU}} \lambda_{f}}}-\frac{\alpha_{2} \rho_{s} \lambda_{h_{2}}}{\alpha_{2} \rho_{s} \lambda_{h_{2}}-\rho_{\mathrm{RSU}} \lambda_{f}} \mathrm{e}^{-\frac{y}{\alpha_{2} \rho_{s} \lambda_{2}}}, \\
f_{Y}(y)=\frac{\mathrm{e}^{-\frac{y}{\rho_{\mathrm{RSU} \lambda_{f}}}}}{\rho_{\mathrm{RSU}} \lambda_{f}-\lambda_{h_{2}} v_{2} \rho_{s}}+\frac{\mathrm{e}^{-\frac{y}{\lambda_{h_{2}} v_{2} \rho_{s}}}}{\lambda_{h_{2}} v_{2} \rho_{s}-\rho_{\mathrm{RSU}} \lambda_{f}} .
\end{gathered}
$$

$\mathrm{OP} \Sigma_{\Sigma_{1} 1}^{\mathrm{FD}}$ can be computed as 


$$
\begin{aligned}
\mathrm{OP}_{\Sigma_{1} 1}^{\mathrm{FD}} & =E_{Y}\left\{1-2 \mathrm{e}^{-\vartheta_{1} Y-\vartheta_{2}} \sqrt{\vartheta_{3} Y+\vartheta_{4}} K_{1}\left(2 \sqrt{\vartheta_{3} Y+\vartheta_{4}}\right)\right\} \\
& =1-\frac{2 \mathrm{e}^{-\vartheta_{2}}}{\rho_{\mathrm{RSU}} \lambda_{f}-\lambda_{h_{2}} v_{2} \rho_{s}} \theta_{1}-\frac{2 \mathrm{e}^{-\vartheta_{2}}}{\lambda_{h_{2}} v_{2} \rho_{s}-\rho_{\mathrm{RSU}} \lambda_{f}} \theta_{2}
\end{aligned}
$$

where $\tau_{1}=\left(\frac{1}{\rho_{R S U} \lambda_{f}}+\vartheta_{1}\right)$,

$$
\begin{aligned}
\theta_{1} & \triangleq \int_{0}^{\infty} \mathrm{e}^{-\tau_{1} y} \sqrt{\vartheta_{3} y+\vartheta_{4}} K_{1}\left(2 \sqrt{\vartheta_{3} y+\vartheta_{4}}\right) d y \\
& =\mathrm{e}^{\frac{\tau_{1} \vartheta_{4}}{\vartheta_{3}}}\left(\frac{\vartheta_{3}}{2 \tau_{1}^{2}} \mathrm{e}^{\frac{\vartheta_{3}}{\tau_{1}}} \Gamma\left(-1, \frac{\vartheta_{3}}{\tau_{1}}\right)-\frac{1}{2} \sum_{m=0}^{M} \frac{\left(-\tau_{1}\right)^{m} \vartheta_{4}^{m+1}}{\vartheta_{3}{ }^{m+1}} G_{1,3}^{2,1}\left(\left.\vartheta_{4}\right|_{1,0,-m} ^{-m}\right)\right), .
\end{aligned}
$$

By using the lase equation in [25], vol. 4, (3.16.2.4)], we have:

$$
\begin{aligned}
\tau_{2} & \triangleq \int_{0}^{\infty} \mathrm{e}^{-\tau_{1} y} \sqrt{\vartheta_{3} y} K_{1}\left(2 \sqrt{\vartheta_{3} y}\right) d y \\
& =\frac{\vartheta_{3}}{2 \tau_{1}^{2}} \mathrm{e}^{\frac{\vartheta_{3}}{\tau_{1}}} \Gamma\left(-1, \frac{\vartheta_{3}}{\tau_{1}}\right) .
\end{aligned}
$$

and $\tau_{3}=\frac{1}{\vartheta_{3}} \int_{0}^{\vartheta_{4}} \mathrm{e}^{-\frac{\tau_{1}}{\vartheta_{3}} y} \sqrt{y} K_{1}(2 \sqrt{y}) d y$. Putting $t=C y \rightarrow y=\vartheta_{4} t \rightarrow d y=\vartheta_{4} d t$ when $y=0 \rightarrow t=0$, $y=\vartheta_{4} \rightarrow t=1 \rightarrow C=\frac{1}{\vartheta_{4}}$. Based on (18) we have:

$$
\begin{aligned}
\tau_{3} & =\frac{\vartheta_{4}}{\vartheta_{3}} \int_{0}^{1} \mathrm{e}^{-\frac{\tau_{1} \vartheta_{4}}{\vartheta_{3}} t} \sqrt{\vartheta_{4} t} K_{1}\left(2 \sqrt{\vartheta_{4} t}\right) d t \\
& =\frac{1}{2} \sum_{m=0}^{M} \frac{\left(-\tau_{1}\right)^{m} \vartheta_{4}^{m+1}}{\vartheta_{3}^{m+1}} G_{1,3}^{2,1}\left(\left.\vartheta_{4}\right|_{1,0,-m} ^{-m}\right) .
\end{aligned}
$$

Where the lase equation follows the fact that $e^{x}=\sum_{k=0}^{\infty} \frac{x^{k}}{k !}$ in [24], (1.211.1)] and $\int_{0}^{1} x^{\lambda}(1-x)^{\mu-1} K_{\nu}(a \sqrt{x}) d x=$ $\frac{2^{\nu-1}}{a^{\nu}} \Gamma(\mu) G_{1,3}^{2,1}\left(\left.\frac{a^{2}}{4}\right|_{\nu, 0, \frac{\nu}{2}-\lambda-\mu} ^{\frac{\nu}{2}-\lambda}\right)$ in [24], (6.952.2)].

And

$$
\begin{aligned}
\theta_{2} & \triangleq \int_{0}^{\infty} \mathrm{e}^{-\tau_{4} y} \sqrt{\vartheta_{3} y+\vartheta_{4}} K_{1}\left(2 \sqrt{\vartheta_{3} y+\vartheta_{4}}\right) d y \\
& =\mathrm{e}^{\frac{\tau_{4} \vartheta_{4}}{\vartheta_{3}}}\left(\frac{\vartheta_{3}}{2 \tau_{4}^{2}} \mathrm{e}^{\frac{\vartheta_{3}}{\tau_{4}}} \Gamma\left(-1, \frac{\vartheta_{3}}{\tau_{4}}\right)-\frac{1}{2} \sum_{m=0}^{M} \frac{\left(-\tau_{4}\right)^{m} \vartheta_{4}^{m+1}}{\vartheta_{3}^{m+1}} G_{1,3}^{2,1}\left(\left.\vartheta_{4}\right|_{1,0,-m} ^{-m}\right)\right) .
\end{aligned}
$$


where $\tau_{4}=\frac{1}{\lambda_{h_{2}} \alpha_{2} \rho_{s}}+\vartheta_{1}$. By using the equation $x^{\nu / 2} K_{\nu}(a \sqrt{x})=\frac{\Gamma(\nu+1)}{(2 p)^{\nu+1}} \mathrm{e}^{\frac{a^{2}}{4 p}} \Gamma\left(-\nu, \frac{a^{2}}{4 p}\right)$ in [25], vol. 4, eq. (3.16.2.4)], we can computed $\tau_{5}$ :

$$
\begin{gathered}
\tau_{5} \triangleq \int_{0}^{\infty} \mathrm{e}^{-\tau_{4} y} \sqrt{\vartheta_{3} y} K_{1}\left(2 \sqrt{\vartheta_{3} y}\right) d y \\
=\frac{\vartheta_{3}}{2 \tau_{4}^{2}} \mathrm{e}^{\frac{\vartheta_{3}}{\tau_{4}}} \Gamma\left(-1, \frac{\vartheta_{3}}{\tau_{4}}\right), \\
\tau_{6}=\frac{1}{\vartheta_{3}} \int_{0}^{\vartheta_{4}} \mathrm{e}^{-\frac{\tau_{4}}{\vartheta_{3}} y} \sqrt{y} K_{1}(2 \sqrt{y}) d y \\
=\frac{1}{2} \sum_{m=0}^{M} \frac{\left(-\tau_{4}\right)^{m} \vartheta_{4}^{m+1}}{\vartheta_{3}{ }^{m+1}} G_{1,3}^{2,1}\left(\left.\frac{4 \vartheta_{4}}{4}\right|_{1,0,-m} ^{-m}\right) .
\end{gathered}
$$

\subsubsection{Case 2:}

If $\frac{1}{\rho_{\mathrm{RSU}} \lambda_{f}}-\frac{1}{\lambda_{h_{2}} \alpha_{2} \rho_{s}}=0$, we have

$$
\begin{gathered}
F_{Y}(y)=1-\mathrm{e}^{\frac{-y}{\rho_{\mathrm{RSU}} \lambda_{f}}}-\frac{y}{\rho_{\mathrm{RSU}} \lambda_{f}} \mathrm{e}^{\frac{-y}{\lambda_{h_{2}}{ }^{2} \rho_{s}}}, \\
f_{Y}(y)=\frac{y}{\lambda_{Y} \lambda_{Y}} \mathrm{e}^{\frac{-y}{\lambda_{Y}}} .
\end{gathered}
$$

$\mathrm{OP}_{\Sigma_{1}, 2}^{\mathrm{FD}}$ is given as

$$
\begin{aligned}
\mathrm{OP}_{\Sigma_{1}, 2}^{\mathrm{FD}} & =E_{Y}\left\{1-2 \mathrm{e}^{-\vartheta_{1} Y-\vartheta_{2}} \sqrt{\vartheta_{3} Y+\vartheta_{4}} K_{1}\left(2 \sqrt{\vartheta_{3} Y+\vartheta_{4}}\right)\right\} \\
& =1-\frac{2}{\lambda_{Y} \lambda_{Y}} \int_{0}^{\infty} \mathrm{e}^{-\left(\frac{-1}{\lambda_{Y}}-\vartheta_{1}\right) y-\vartheta_{2}}\left(\frac{1}{\vartheta_{3}}\left(y \vartheta_{3}+\vartheta_{4}\right)-\frac{\vartheta_{4}}{\vartheta_{3}}\right) \sqrt{\vartheta_{3} y+\vartheta_{4}} K_{1}\left(2 \sqrt{\vartheta_{3} y+\vartheta_{4}}\right) d y \\
& =1-\theta_{4}+\theta_{5}
\end{aligned}
$$

where

$$
\begin{aligned}
\theta_{4} & =\tau_{7} \int_{0}^{\infty} \mathrm{e}^{-\tau_{8} y}\left(\vartheta_{3} y+\vartheta_{4}\right)^{3 / 2} K_{1}\left(2 \sqrt{\vartheta_{3} y+\vartheta_{4}}\right) d y \\
& =\tau_{7} \mathrm{e}^{\frac{\tau_{8} \vartheta_{4}}{\vartheta_{3}}}\left(\Delta_{1}-\Delta_{2}\right) \\
& =\tau_{7} \mathrm{e}^{\frac{\tau_{8} \vartheta_{4}}{\vartheta_{3}}}\left[\frac{\vartheta_{3}}{\tau_{8}{ }^{2}} W_{-2,1 / 2}\left(\frac{\vartheta_{3}}{\tau_{8}}\right)-\frac{1}{2} \sum_{m=0}^{M} \frac{\left(-\tau_{8}\right)^{m} \vartheta_{4}{ }^{m+2}}{\vartheta_{3}{ }^{m+1}} G_{1,3}^{2,1}\left(\left.\vartheta_{4}\right|_{1,0,-m} ^{-m}\right)\right]
\end{aligned}
$$

By using the equation $\int_{0}^{\infty} x^{\nu / 2} K_{\nu}(a \sqrt{x}) d x=\frac{p^{-\mu-1 / 2}}{a} \Gamma\left(\mu+\frac{\nu}{2}+1\right) \Gamma\left(\mu-\frac{\nu}{2}+1\right) \exp \left(\frac{a^{2}}{8 p}\right) \times W_{-\mu-1 / 2, \nu / 2}\left(\frac{a^{2}}{4 p}\right)$ in [25], vol. 4, eq. (3.16.2.3)] and $\int_{0}^{\infty} x^{\nu / 2} K_{\nu}(a \sqrt{x}) d x=\frac{\Gamma(\nu+1)}{(2 p)^{\nu+1}} \exp \left(\frac{a^{2}}{4 p}\right) \Gamma\left(-\nu, \frac{a^{2}}{4 p}\right)$ in [25], vol. 4, eq. (3.16.2.4)], we can computed $\Delta_{1}, \Delta_{2}^{0}$ :

$$
\begin{aligned}
\Delta_{1} & =\int_{0}^{\infty} \mathrm{e}^{-\tau_{8} y} \vartheta_{3}^{3 / 2} y^{3 / 2} K_{1}\left(2 \sqrt{\vartheta_{3} y}\right) d y \\
& =\frac{\vartheta_{3}}{\tau_{8}^{2}} W_{-2,1 / 2}\left(\frac{\vartheta_{3}}{\tau_{8}}\right),
\end{aligned}
$$




$$
\begin{aligned}
\Delta_{2} & =\frac{1}{\vartheta_{3}} \int_{0}^{\vartheta_{4}} \mathrm{e}^{-\frac{\tau_{8}}{\vartheta_{3}} y} y^{3 / 2} K_{1}(2 \sqrt{y}) d y \\
& =\frac{1}{2} \sum_{m=0}^{M} \frac{\left(-\tau_{8}\right)^{m} \vartheta_{4}^{m+2}}{\vartheta_{3}{ }^{m+1}} G_{1,3}^{2,1}\left(\left.\vartheta_{4}\right|_{1,0,-m} ^{-m}\right),
\end{aligned}
$$

and

$$
\begin{aligned}
\theta_{5} & =\tau_{7} \vartheta_{4} \int_{0}^{\infty} \mathrm{e}^{-\tau_{8} y} \sqrt{\vartheta_{3} y+\vartheta_{4}} K_{1}\left(2 \sqrt{\vartheta_{3} y+\vartheta_{4}}\right) d y \\
& =\mathrm{e}^{\frac{\tau_{8} \vartheta_{4}}{\vartheta_{3}}}\left(\frac{\tau_{7} \vartheta_{4} \vartheta_{3}}{2 \tau_{8}^{2}} \mathrm{e}^{\frac{\vartheta_{3}}{\tau_{8}}} \Gamma\left(-1, \frac{\vartheta_{3}}{\tau_{8}}\right)-\frac{\tau_{7}}{2} \sum_{m=0}^{M} \frac{\left(-\tau_{8}\right)^{m} \vartheta_{4}^{m+2}}{\vartheta_{3}^{m+1}} G_{1,3}^{2,1}\left(\left.\vartheta_{4}\right|_{1,0,-m} ^{-m}\right)\right)
\end{aligned}
$$

where

$$
\tau_{7}=\frac{2 \mathrm{e}^{-\vartheta_{2}}}{\lambda_{Y} \lambda_{Y} \vartheta_{3}}, \tau_{8}=\frac{1}{\lambda_{Y}}+\vartheta_{1}, \quad \tau_{9}=\frac{\vartheta_{3}}{2 \tau_{8}^{2}} \mathrm{e}^{\frac{\vartheta_{3}}{\tau_{8}}} \Gamma\left(-1, \frac{\vartheta_{3}}{\tau_{8}}\right), \tau_{10}=\frac{1}{2} \sum_{m=0}^{M} \frac{\left(-\tau_{8}\right)^{m} \vartheta_{4} m+1}{\vartheta_{3} m+1} G_{1,3}^{2,1}\left(\left.\frac{4 \vartheta_{4}}{4}\right|_{1,0,-m} ^{-m}\right) .
$$

\subsection{The outage probability for group 2}

The exact outage probability of group 2 can be written as

$$
\mathrm{OP}_{\Sigma_{2}}^{\mathrm{FD}}=1-\operatorname{Pr}\left(\gamma_{\mathrm{U}_{2} \leftarrow 1} \geq \gamma_{0}^{1}, \gamma_{\mathrm{U}_{2}} \geq \gamma_{0}^{2}\right)
$$

The outage probability in (29) can be computed as

$$
\begin{aligned}
\mathrm{OP}_{\Sigma_{2}}^{\mathrm{FD}} & =1-\operatorname{Pr}\left(\begin{array}{l}
\frac{\alpha_{1} \rho_{\mathrm{RSU}} \rho_{s}\left|g_{2}\right|^{2}\left|h_{1}\right|^{2}}{\alpha_{2} \rho_{\mathrm{RSU}} \rho_{s}\left|h_{2}\right|^{2}\left|g_{2}\right|^{2}+\rho_{\mathrm{RSU}}\left|g_{2}\right|^{2}+\alpha_{1} \rho_{s}\left|h_{1}\right|^{2}+\rho_{\mathrm{RSU}} \rho_{\mathrm{RSU}}|f|^{2}\left|g_{2}\right|^{2}+1} \geq \gamma_{0}^{1} \\
\frac{\alpha_{2} \rho_{\mathrm{RSU}} \rho_{s}\left|h_{2}\right|^{2}\left|g_{2}\right|^{2}}{\rho_{\mathrm{RSU}}\left|g_{2}\right|^{2}+\alpha_{1} \rho_{s}\left|h_{1}\right|^{2}+\rho_{\mathrm{RSU}} \rho_{\mathrm{RSU}}|f|^{2}\left|g_{2}\right|^{2}+1} \geq \gamma_{0}^{2}
\end{array}\right) \\
& =1-\left(\mathrm{OP}_{\Sigma_{2}, 1}^{\mathrm{FD}}+\mathrm{OP}_{\Sigma_{2}, 2}^{\mathrm{FD}}\right) .
\end{aligned}
$$

\subsubsection{Case 1:}

If $\max =\frac{1}{\frac{\alpha_{1} \rho_{s}}{\gamma_{0}^{1}}\left|h_{1}\right|^{2}-\alpha_{2} \rho_{s}\left|h_{2}\right|^{2}-1-\rho_{\mathrm{RSU}}|f|^{2}}$, we have:

$$
\begin{aligned}
& \mathrm{OP}_{\Sigma_{2}, 1}^{\mathrm{FD}}=\int_{0}^{\infty} f_{|f|^{2}}(z) d z \int_{\substack{\gamma_{0}^{2} \\
\alpha_{2} \rho_{s}}}^{\infty} f_{\left|h_{2}\right|^{2}}(y) d y \\
& \times \underbrace{\int_{\frac{\gamma_{0}^{1}}{\alpha_{1} \rho_{s}}\left(\alpha_{2} \rho_{s} y+\rho_{\mathrm{RSU}} z+1\right)}^{\frac{\gamma_{0}^{1} \alpha_{2}}{\alpha_{1}}\left(\frac{1}{\gamma_{0}^{2}}+1\right) y}\left(1-F_{\left|g_{2}\right|^{2}}\left(\frac{1}{\rho_{\mathrm{RSU}}} \frac{\alpha_{1} \rho_{s} x+1}{\frac{\alpha_{1} \rho_{s}}{\gamma_{0}^{1}} x-\alpha_{2} \rho_{s} y-\rho_{\mathrm{RSU}} z-1}\right)\right) f_{\left|h_{1}\right|^{2}}(x) d x}_{\triangleq_{\Delta_{4}}},
\end{aligned}
$$


Continuing the calculation process, we have

$$
\begin{aligned}
& \mathrm{OP}_{\Sigma_{2}, 1}^{\mathrm{FD}}=\int_{0}^{\infty} f_{|f|^{2}}(z) d z[\underbrace{\frac{1}{\lambda_{h_{2}}} \int_{\frac{\gamma_{0}^{2}}{\alpha_{2} \rho_{s}}\left(\rho_{\mathrm{RSU}} z+1\right)}^{\infty} \mathrm{e}^{-\frac{\gamma_{0}^{1}}{\rho_{\mathrm{RSU} g_{2}}}} \mathrm{e}^{-\left(\frac{\gamma_{0}^{1} \alpha_{2}}{\alpha_{1} h_{1}}\left(\frac{1}{\gamma_{0}^{2}}+1\right)+\frac{1}{\lambda_{h_{2}}}\right) y} d y}_{\triangleq_{\vartheta_{1}}}
\end{aligned}
$$

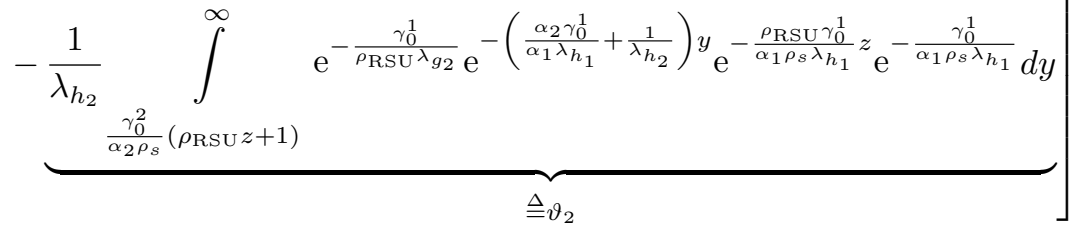

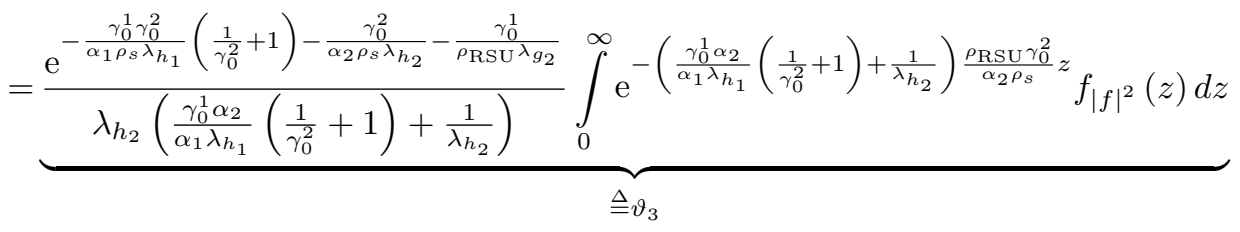

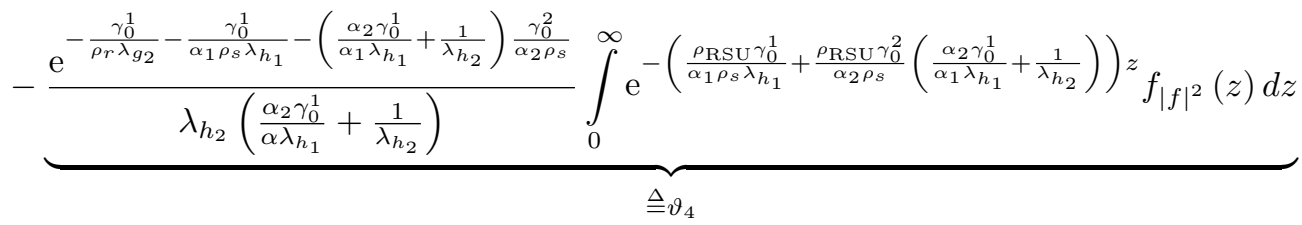

$$
\begin{aligned}
& =\frac{\mathrm{e}^{-\frac{\gamma_{0}^{1} \gamma_{0}^{2}}{\alpha_{1} \rho_{s} h_{1}}\left(\frac{1}{\gamma_{0}^{2}}+1\right)-\frac{\gamma_{0}^{2}}{\alpha_{2} \rho_{s} \lambda h_{2}}-\frac{\gamma_{0}^{1}}{\rho_{\mathrm{RSU}} g_{2}}}}{\lambda_{h_{2}} \lambda_{f}\left(\frac{\gamma_{0}^{1} \alpha_{2}}{\alpha_{1} \lambda_{h_{1}}}\left(\frac{1}{\gamma_{0}^{2}}+1\right)+\frac{1}{\lambda_{h_{2}}}\right)\left[\left(\frac{\gamma_{0}^{1} \alpha_{2}}{\alpha_{1} \lambda_{h_{1}}}\left(\frac{1}{\gamma_{0}^{2}}+1\right)+\frac{1}{\lambda_{h_{2}}}\right) \frac{\rho_{\mathrm{RSU}} \gamma_{0}^{2}}{\alpha_{2} \rho_{s}}+\frac{1}{\lambda_{f}}\right]} \\
& -\frac{\mathrm{e}^{-\frac{\gamma_{0}^{1}}{\rho_{\mathrm{RSU} g_{2}}}-\frac{\gamma_{0}^{1}}{\alpha_{1} \rho_{s} \lambda_{1}}-\left(\frac{\alpha_{2} \gamma_{0}^{1}}{\alpha_{1} \lambda_{1}}+\frac{1}{\lambda_{h_{2}}}\right) \frac{\gamma_{0}^{2}}{\alpha_{2} \rho_{s}}}}{\lambda_{h_{2}} \lambda_{f}\left(\frac{\alpha_{2} \gamma_{0}^{1}}{\alpha_{1} \lambda_{h_{1}}}+\frac{1}{\lambda_{h_{2}}}\right)\left[\left(\frac{\rho_{\mathrm{RSU}} \gamma_{0}^{1}}{\alpha_{1} \rho_{s} \lambda_{h_{1}}}+\frac{\rho_{r} \gamma_{0}^{2}}{\alpha_{2} \rho_{s}}\left(\frac{\alpha_{2} \gamma_{0}^{1}}{\alpha_{1} \lambda_{h_{1}}}+\frac{1}{\lambda_{h_{2}}}\right)\right)+\frac{1}{\lambda_{f}}\right]} .
\end{aligned}
$$

$$
\begin{aligned}
& \text { where } \Delta_{4}=\frac{1}{\lambda_{h_{1}}} \mathrm{e}^{-\frac{\gamma_{0}^{1}}{\rho_{\mathrm{RSU}} g_{2}}} \quad \int_{1}^{\frac{\gamma_{0}^{1} v_{2}}{v_{1}}\left(\frac{1}{\gamma_{0}^{2}}+1\right) y} \mathrm{e}^{-\frac{x}{\lambda_{h_{1}}}} d x \text {, } \\
& \frac{\gamma_{0}^{1}}{v_{1} \rho_{s}}\left(v_{2} \rho_{s} y+\rho_{\mathrm{RSU}} z+1\right)
\end{aligned}
$$

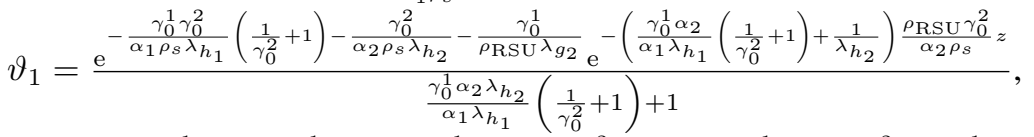

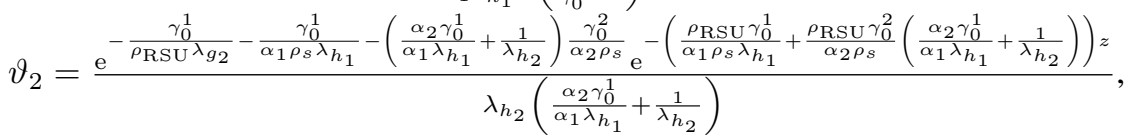

$$
\begin{aligned}
& \vartheta_{3}=\frac{\mathrm{e}^{-\frac{\gamma_{0}^{1} \gamma_{0}^{2}}{\alpha_{1} \rho_{s} \lambda h_{1}}\left(\frac{1}{\gamma_{0}^{2}}+1\right)-\frac{\gamma_{0}^{2}}{\alpha_{2} \rho_{s} \lambda h_{2}}-\frac{\gamma_{0}^{1}}{\rho_{\mathrm{RSU}} g_{2}}}}{\lambda_{h_{2}} \lambda_{f}\left(\frac{\gamma_{0}^{1} \alpha_{2}}{\alpha_{1} h_{1}}\left(\frac{1}{\gamma_{0}^{2}}+1\right)+\frac{1}{\lambda_{h_{2}}}\right)\left[\left(\frac{\gamma_{0}^{1} \alpha_{2}}{\alpha_{1} \lambda_{1}}\left(\frac{1}{\gamma_{0}^{2}}+1\right)+\frac{1}{\lambda_{h_{2}}}\right) \frac{\rho_{\mathrm{RSU}} \gamma_{0}^{2}}{\alpha_{2} \rho_{s}}+\frac{1}{\lambda_{f}}\right]}, \\
& \vartheta_{4}=\frac{\mathrm{e}^{-\frac{\gamma_{0}^{1}}{\rho_{\mathrm{RSU}} g_{2}}-\frac{\gamma_{0}^{1}}{\alpha_{1} \rho_{s} h_{1}}-\left(\frac{\alpha_{2} \gamma_{0}^{1}}{\alpha_{1} h_{1}}+\frac{1}{\lambda_{h_{2}}}\right) \frac{\gamma_{0}^{2}}{\alpha_{2} \rho_{s}}}}{\lambda_{h_{2}} \lambda_{f}\left(\frac{\alpha_{2} \gamma_{0}^{1}}{\alpha_{1} \lambda h_{1}}+\frac{1}{\lambda_{h_{2}}}\right)\left[\left(\frac{\rho_{\mathrm{RSU}} \gamma_{0}^{1}}{\alpha_{1} \rho_{s} h_{1}}+\frac{\rho_{\mathrm{RSU}} \gamma_{0}^{2}}{\alpha_{2} \rho_{s}}\left(\frac{\alpha_{2} \gamma_{0}^{1}}{\alpha_{1} \lambda_{h_{1}}}+\frac{1}{\lambda_{h_{2}}}\right)\right)+\frac{1}{\lambda_{f}}\right]} .
\end{aligned}
$$




\subsubsection{Case 2:}

If $\max =\frac{1}{\frac{\alpha_{2} \rho_{s}}{\gamma_{0}^{2}}\left|h_{2}\right|^{2}-1-\rho_{\mathrm{RSU}}|f|^{2}}$, we have:

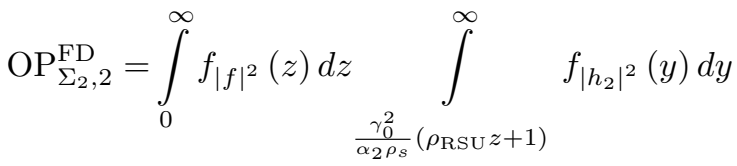

$$
\begin{aligned}
& \times \underbrace{\int_{\frac{\gamma_{0} \alpha_{2}}{\alpha_{1}}\left(\frac{1}{\gamma_{0}^{2}}+1\right) y}^{\infty}\left(1-F_{\left|g_{2}\right|^{2}}\left(\frac{1}{\rho_{\mathrm{RSU}}} \frac{\alpha_{1} \rho_{s} x+1}{\frac{\alpha_{2} \rho_{s}}{\gamma_{0}^{2}} y-\rho_{\mathrm{RSU}} z-1}\right)\right) f_{\left|h_{1}\right|^{2}}(x) d x}_{\triangleq \Delta_{3}} \\
& =\frac{2 \gamma_{0}^{2}}{\alpha_{2} \rho_{s} \rho_{\mathrm{RSU}} \lambda_{f}} \sum_{k=1}^{\infty} k \lambda_{g_{2}}^{k}\left(-\alpha_{1} \rho_{s} \lambda_{h_{1}}\right)^{k-1} e^{-\Xi_{4}-\frac{\gamma_{0}^{2}}{\alpha_{2} \rho_{s} h_{2}}} \sqrt{\left(\frac{\Xi_{2}(1+E)}{\Xi_{1} \lambda_{g_{2}}}\right)^{k+1}\left(\frac{\rho_{\mathrm{RSU}} \Xi_{2} \Xi_{5}}{\Xi_{1} \lambda_{g_{2}}}\right)^{k+1}} \\
& \times \int_{0}^{\infty} \mathrm{e}^{-\left(\frac{1}{\lambda_{f}}+\frac{\Xi_{1} \rho_{\mathrm{RSU}} \rho_{\mathrm{RSU}}}{\Xi_{2}}+\frac{\rho_{\mathrm{RSU}} \gamma_{0}^{2}}{\alpha_{2} \rho_{s} \lambda h_{2}}\right) z} z^{k+1} K_{k+1}\left(2 \sqrt{\frac{\Xi_{1}\left(1+\rho_{\mathrm{RSU}} \Xi_{5} z+\Xi_{5}\right)}{\Xi_{2} \lambda_{g_{2}}}}\right) d z .
\end{aligned}
$$

where $\Xi_{1}=\frac{\gamma_{0}^{1} \alpha_{2}}{\lambda_{h_{1}} \alpha_{1}}\left(\frac{1}{\gamma_{0}^{2}}+1\right), \Xi_{2}=\frac{\alpha_{2} \rho_{s} \rho_{\mathrm{RSU}}}{\gamma_{0}^{2}}, \Xi_{4}=\frac{\rho_{s} \lambda_{h_{1}} \alpha_{1} \Xi_{1}}{\lambda_{g_{2}} \Xi_{2}}+\frac{\Xi_{1} \rho_{\mathrm{RSU}}}{\Xi_{2}}, \Xi_{5}=\frac{\rho_{s} \rho_{\mathrm{RSU}} \lambda_{h_{1}} \alpha_{1} \Xi_{1}}{\Xi_{2}}$.

\subsection{Throughput}

The throughput in delay-limited transmission mode is given by

$$
\begin{aligned}
& \Upsilon_{1}=\left(1-O P_{\Sigma_{1}}^{\mathrm{FD}}\right) \mathrm{R}_{1}, \\
& \Upsilon_{2}=\left(1-O P_{\Sigma_{2}}^{\mathrm{FD}}\right) \mathrm{R}_{2} .
\end{aligned}
$$

\section{RESULT AND DISCUSSION}

Figures 2 and 3 demonstrate outage probability of two vehicles. It can be observed that outage probability improves significantly at high SNR. There is existence performance gap among two vehicles due to different power allocation factors. It is further confirmed that outage probability cannot improve at very high SNR regardless of changing target rates $R_{1}, R_{2}$. In these simulations, Monte-Carlo and analytical simulations are matched tightly and it confirmed our derivations are correct. Figure 4 examines impact of interference channel related to FD mode on outage probability. When changing $\lambda_{f}$ from $0(\mathrm{~dB})$ to $30(\mathrm{~dB})$ outage probability becomes worse significantly. Figure 5 shows throughput versus transmit SNR $\rho$. Increasing $\rho$ from $-20(\mathrm{~dB})$ to $15(\mathrm{~dB})$, throughput increases significantly, but it meets the ceiling at high SNR region.

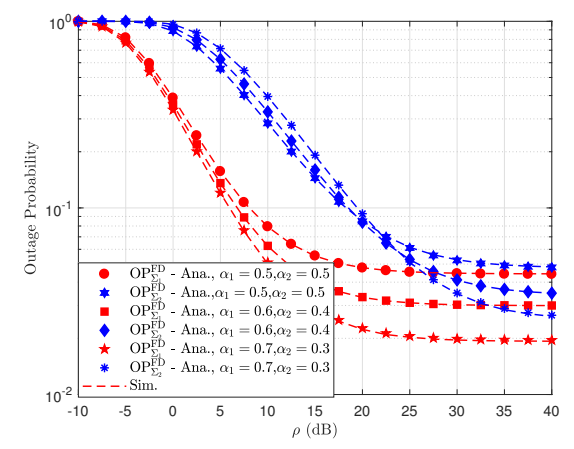

Figure 2. Outage probability: $\mathrm{R}_{1}=0.5$ $\mathrm{bps} / \mathrm{Hz}, \mathrm{R}_{2}=1 \mathrm{bps} / \mathrm{Hz}$.

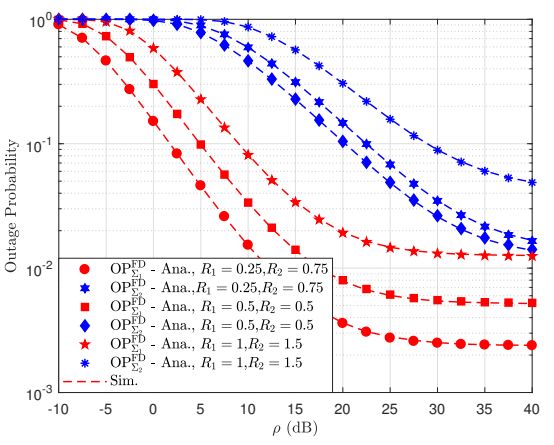

Figure 3. Outage probability: $\alpha_{1}=0.9$, $\alpha_{2}=0.1$ 


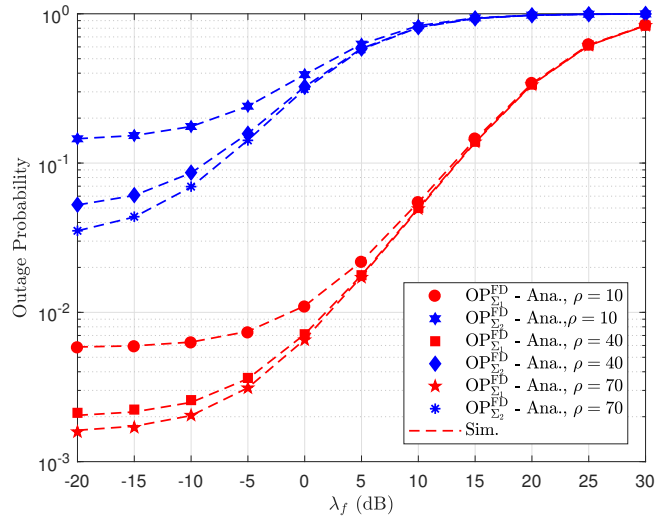

Figure 4. Outage probability: $\mathrm{R}_{1}=0.1 \mathrm{bps} / \mathrm{Hz}$ $\mathrm{R}_{2}=0.1, \alpha_{1}=0.9, \alpha_{2}=0.1$.

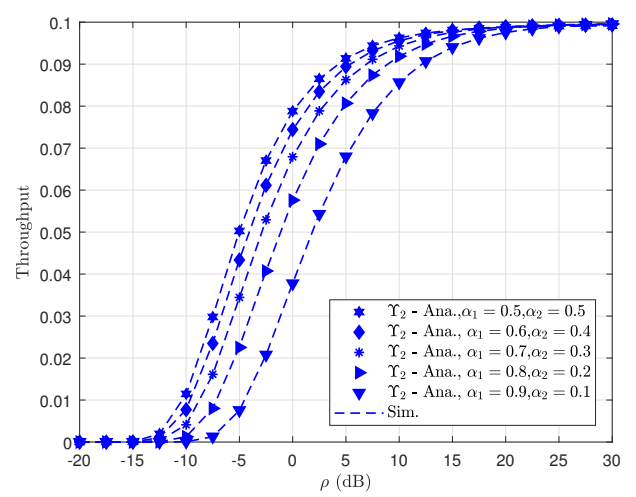

Figure 5. Throughput: $\alpha_{1}=0.9, \alpha_{2}=0.1$.

\section{CONCLUSION}

In this paper, two pairs of users in NOMA-V2X systems have been proposed for $5 \mathrm{G}$ cellular V2X communications. The fixed power allocation factors are applied to highlight different outage performance of each group of user. We provided exact expressions of outage probability to evaluate system performance. We show that the formulated expression is verified via simulations. Fortunately, it indicates reasonable performance of two vehicles in NOMA-V2X if self-interference channel is controlled well. Simulation results demonstrate that the proposed scheme exhibits better performance at high SNR at sources.

\section{REFERENCES}

[1] Z. Ding, et al., "A Survey on Non-Orthogonal Multiple Access for 5G Networks: Research Challenges and Future Trends," IEEE J. Sel. Areas Commun., vol. 35, no. 10, pp. 2181-2195, Oct. 2017.

[2] T.-L. Nguyen and Dinh-Thuan Do, "Exploiting Impacts of Intercell Interference on SWIPT-assisted Nonorthogonal Multiple Access," Wireless Communications and Mobile Computing, 2018.

[3] F. Liu, et al., "Proportional fairness-based user pairing and power allocation for non-orthogonal multiple access," Proc. IEEE PIMRC., pp. 1127-1131, 2015.

[4] X.-X. Nguyen and Dinh-Thuan Do, "Maximum Harvested Energy Policy in Full-Duplex Relaying Networks with SWIPT," in International Journal of Communication Systems (Wiley), vol. 30, no. 17, pp. 2181-2195, Jul. 2017.

[5] Dinh-Thuan Do, et al., "Wireless powered relaying networks under imperfect channel state information: system performance and optimal policy for instantaneous rate," Radioengineering, vol. 26, no. 3, pp. 869-877, Sep. 2017.

[6] X.-X. Nguyen and Dinh-Thuan Do, "Optimal power allocation and throughput performance of full-duplex DF relaying networks with wireless power transfer-aware channel," EURASIP Journal on Wireless Communications and Networking, Sep. 2017.

[7] T.-L. Nguyen; Dinh-Thuan Do, "A new look at AF two-way relaying networks: energy harvesting architecture and impact of co-channel interference," Annals of Telecommunications, vol. 72, no. 11, pp. 669-678, Dec. 2017.

[8] Dinh-Thuan Do and C.-B. Le, "Application of NOMA in Wireless System with Wireless Power Transfer Scheme: Outage and Ergodic Capacity Performance Analysis," in Sensors, vol. 18, no. 10, pp. 669-678, Oct. 2018.

[9] M. F. Kader, et al., "Full-Duplex Non-Orthogonal Multiple Access in Cooperative Relay Sharing for 5G Systems," IEEE Transactions on Vehicular Technology, vol. 67, no. 7, pp. 5831-5840, July 2018, doi: 10.1109/TVT.2018.2799939.

[10] Dinh-Thuan Do, C.-B. Le, "Exploiting Outage Performance of Wireless Powered NOMA,” TELKOMNIKA Telecommunication, Computing, Electronics and Control, vol. 16, no. 5, pp. 1907-1917, 2018. 
[11] D. Chowdhury, et al., "Design and implementation of a grid-tied emergency back-up power supply for medium power applications," International Journal of Electrical and Computer Engineering (IJECE), ISSN 2088-8708, doi: http://doi.org/10.11591/ijece.v10i6.pp

[12] Dinh-Thuan Do, et al.," "Cooperative underlay cognitive radio assisted NOMA: secondary network improvement and outage performance," TELKOMNIKA Telecommunication, Computing, Electronics and Control, vol. 17, no. 5, pp. 2147-2154, 2019.

[13] Dinh-Thuan Do, T.-T. Thi Nguyen, "Exact Outage Performance Analysis of Amplify and Forward-Aware Cooperative NOMA," TELKOMNIKA Telecommunication, Computing, Electronics and Control, vol. 16, no. 5, pp. 1966-1973, 2018.

[14] J. Guo, et al., "User pairing and power allocation for downlink non-orthogonal multiple access," Proc. IEEE Globecom Workshops, Dec. 2018.

[15] H. Zhang, et al., "User pairing algorithm with SIC in non-orthogonal multiple access system," Proc. Int. Conf.Commun., May. 2016.

[16] T. Sahin, et al., "Virtual cells for 5G V2X communications," IEEE Commun. Standards Mag., vol. 2, no. 1, pp. 22-28, Mar. 2018.

[17] C. Backfrieder, et al., "Increased traffic flow through node-based bottleneck prediction and V2X communication,” in IEEE Trans. Intell. Transp. Syst., vol. 18, no. 2, pp. 349-363, Feb. 2017.

[18] K. Lee, et al., "Latency of cellular-based V2X: Perspectives on TTI-proportional latency and TTIindependent latency," IEEE Access, vol. 5, pp. 15800-15809, 2017.

[19] R. M. Masegosa and J. Gozalvez, "LTE-V for sidelink 5G V2X vehicular communications: A new 5G technology for short-range vehicle-to-everything communications," IEEE Veh. Technol. Mag., vol. 12, no. 4, pp. 30-39, Dec. 2017.

[20] S. Chen, et al., "Vehicleto-everything (V2X) services supported by LTE-based systems and 5G," IEEE Commun. Standards Mag., vol. 1, no. 2, pp. 70-76, Jun. 2017.

[21] H. Ullah, et al., "5G communication: An overview of vehicle-to-everything, drones, and healthcare usecases," in IEEE Access, vol. 7, pp. 37251-37268, 2019.

[22] XB. W. Khoueiry and M. R. Soleymani, "An efficient NOMA V2X communication scheme in the Internet of vehicles," Proc. VTC-Spring, Sydney, NSW, Australia, pp. 1-7, Jun. 2017.

[23] C. B. Math, et al., "V2X applicationreliability analysis of data-rate and message-rate congestion control algorithms," IEEE Commun. Lett., vol. 21, no. 6, pp. 1285-1288, Jun. 2017.

[24] S. D. Gradshteyn, et al., "Table of Integrals, Series, and Products. Mathematics of Computation," vol. 20, no. 96, pp. 616. doi:10.2307/2003554.

[25] A., P., Pridnikov, et al., ’Integrals and Series: Direct Laplace transforms,” Jan. 1992. 